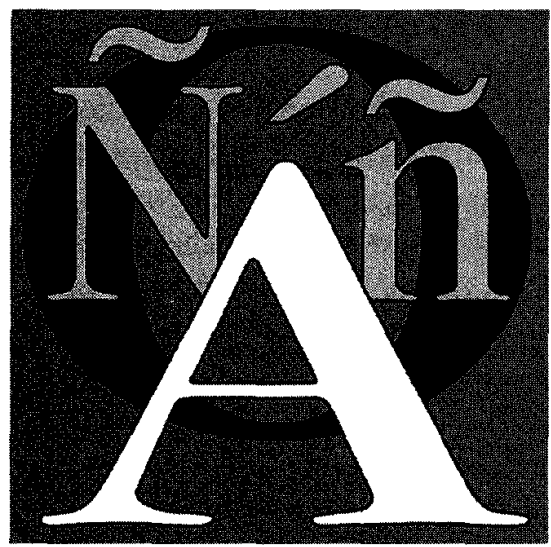

\title{
Una aventura didáctica por internet
}

CRISTINA Simón Ruiz

\section{INTRODUCCIÓN}

Cuando el año pasado la profesora Emma Jiménez me invitó a participar en esta aventura, acepté casi de inmediato, aunque algunas dudas sobre los resultados que obtendríamos me atenazaban los nervios.

La idea original era poner en contacto, vía intemet, a mis estudiantes de redacción en español, segunda lengua (L2), nivel III, con los estudiantes de la profesora Emma para que compartieran parte de su proceso de adquisición y reflexionaran sobre él, sobre todo en lo que respecta a las diferencias entre adquirir una L2 y una lengua extranjera (L3). Sin embargo, sufrimos una primera decepción, pues mis estudiantes, aduciendo sobre todo el hecho de no tener un acceso personal a la red en México, rechazaron la invitación.

Entonces, surgió la idea de virar nuestros planteamientos iniciales y poner en contacto a los alumnos del curso de Formación de Profesores de Español como L2 o LE con los estudiantes canadienses y, adelantándome a las conclusiones, puedo decir que los resultados son alentadores.

En primer lugar, hay que aclarar que los objetivos iniciales cambiaron, obviamente. Lo que decidimos lograr fue, por una parte, que los futuros profesores de español como L2/E empezaran a reflexionar sobre los problemas de adquisición del español como LE por un grupo de alumnos bastante homogéneo y con una misma lengua materna: el francés. Por otra parte, pretendíamos que los alumnos canadienses establecieran un contacto directo con hispanohablantes que no fueran sus profesoras de español y, a través de un contacto directo y personal, tuvieran la oportunidad de reflexionar sobre algunos de sus problemas con la adquisición del español, tanto en el plano gramatical como en el sociocultural. 


\section{DESCRIPCIÓN DE LOS PARTICIPANTES DE LA AVENTURA}

Los estudiantes-profesores que siguen el curso que imparte el CEPE no son especialistas en la materia, sino, principalmente, personas que extraoficialmente ya han enfrentado la experiencia de enseñar, o bien alguna lengua extranjera a mexicanos - sobre todo inglés-, o bien español a no hispanohablantes o que vislumbran que, en un futuro próximo, lo harán - ya por motivos de becas fuera del país, ya por otras razones-.

En general son alumnos jóvenes -el promedio oscila entre los 23 y los 35 años-y provienen de muy diversas formaciones - arquitectura, letras modernas, diseño gráfico, pedagogía, música por especificar las más generales-, o bien, han concluido recientemente sus estudios de bachillerato, por lo que el curso que se les imparte —que tiene un año de duración — busca, principalmente, darles las bases más importantes para enfrentar este reto con la mejor preparación posible.

Sin embargo, debido a las normas internas del CEPE, estos estudiantes no pueden practicar sus conocimientos con los grupos de aprendices extranjeros que se forman en nuestras aulas, lo que los priva de esa necesaria experiencia directa para poner en práctica los conocimientos adquiridos.

\section{DESCRIPCIÓN DE LOS PASOS DE LA AVENTURA}

De esta manera, cuando mis aprendices extranjeros declinaron participar en la aventura, la profesora Emma y yo vislumbramos la maravillosa posibilidad de poner en contacto a estos dos grupos. Pensamos que, con esta comunicación directa, los estudiantes para ser profesores tendrían la invaluable posibilidad de enfrentar directamente tanto los problemas gramaticales como los culturales con los que topa cualquier persona que quiera apropiarse de una lengua extranjera.

Con este planteamiento inicial, elaboramos un primer calendario de colaboraciones que, aunque no se cumplió cabalmente en cuanto a las fechas y al número total de participantes, sí tuvo una conclusión enriquecedora para ambas partes.

La dinámica fue la siguiente:

1. Se les dio un tiempo razonable para que se enviaran correos electrónicos en los cuales se presentaban a sí mismos y preguntaban a sus destinatarios por sus propias vidas. 
Así lo hicieron y la estrategia que seguimos fue la de que los alumnos mexicanos contestaban a los canadienses sin mencionar explícitamente los errores gramaticales que habían detectado en sus primeros envíos y que entorpecían la comunicación, sino, simplemente, enmendándoles la plana en sus respuestas.

Correspondió a la profesora Emma Jiménez hacer la reflexión explícita en el aula, en donde se explicaron y corrigieron dichos errores.

Por nuestra parte, mis estudiantes sólo subrayaron los errores que detectaron en los correos que recibieron y yo los sistematicé en los rubros correspondientes. ${ }^{1}$ Esta sistematización ha sido entregada a mis alumnos con los que, en varias sesiones de una media hora cada una, reflexionamos punto por punto sobre dichos errores.

2. Se les pidió a ambos grupos que escribieran un brevísimo artículo para la revista Flores de Nieve, que se publica en español en internet, gracias a los esfuerzos de la profesora Emma Jiménez y del licenciado Carlos Negrete. El tema de los artículos era totalmente libre y, una vez escritos, debían ser enviados a los correspondientes destinatarios para que éstos hicieran comentarios, tanto al fondo como a la forma de los mismos, en un plazo de tiempo razonable.

3. Una vez corregidos los errores formales y de contenido, éstos estuvieron listos para ser publicados y han aparecido ya en el número seis de la revista antes mencionada. ${ }^{2}$

4. Elaboramos un brevísimo cuestionario para poder evaluar los resultados de la aventura y se los dimos a los estudiantes de ambos lados para que lo respondieran. ${ }^{3}$

5. Con base en los resultados que hemos obtenido y gracias al entusiasmo de los participantes de ambos lados, reanudaremos la aventura a partir del mes de agosto, tratando de mejorar los puntos débiles de la primera parte de dicha aventura y que aparecen especificados en el último rubro de esta reflexión.

\footnotetext{
${ }^{1}$ Anexo, al final de esta reflexión, dicha sistematización de errores.

${ }^{2}$ Para los lectores que quieran conocer esta publicación, la dirección es la siguiente:

http://www.webruler.com/negrete/jiménez.

${ }^{3}$ Asimismo, anexo el formato de la mencionada encuesta.
} 


\section{DINÁMICA DE TRABAJO}

Desde México, nosotros no tuvimos que dedicar un tiempo excesivo a esta aventura y no por falta de ganas, sino porque ésta fue una actividad extracurricular para los estudiantes-profesores que voluntariamente aceptaron participar.

Tuvimos, aproximadamente, unas diez sesiones de media hora a lo largo de los casi cuatro meses que duró esta experiencia y, en ellas, reflexionábamos someramente, tanto y principalmente sobre los puntos gramaticales problemáticos que ellos encontraban en los correos electrónicos de sus corresponsales, como en sus causas y posibles consecuencias.

Independientemente de que, en varias ocasiones, no sabían nombrarlos, su intuición lingüística como nativohablantes fue suficiente para que los ubicarán cabalmente y para que, comparativamente o no con sus conocimientos del francés, entendieran los motivos de la repetición de los mismos.

Lo que no hicimos en profundidad, tanto por falta de tiempo cuanto porque los estudiantes-profesores tienen dos asignaturas para ello -Metodología y Aspectos Problemáticos del Español-, fue reflexionar y ahondar sobre los caminos metodológicos que nos permiten poner en práctica ciertas soluciones para que este tipo de estudiantes pueda superar con mejor éxito estos inevitables tropezones gramaticales.

Quiero también comentar que, en lo que respecta a la detección de nuestras diferencias culturales y de organización social, los comentarios han sido muy poco significativos. Pienso que esto se debe, tanto al tiempo de vida de esta aventura como a que los temas que hemos tratado son superficiales y no se aprestaron a evidenciar estas diferencias.

\section{CONCLUSIONES}

En este último rubro, me permito mos los resultados del cuestionario que obtuve de mis estudiantes, así como unas consideraciones finales.

En cuanto a las respuestas del cuestionario final de mis estudiantes, creo que son dignos de comentar los siguientes aspectos:

1. La mayoría de ellos, aun cuando ya señalé que fue una actividad extracurricular, demandaron que se dedicara más tiempo al intercambio de correos electrónicos, principalmente, para conocer mejor a sus corresponsales, como para tener más elementos - gramaticales, culturales, sociales- con los que enriquecer la experiencia. 
2. Sugirieron también que hubiera más sesiones extra, para tener la oportunidad de reflexionar con mayor profundidad en los aspectos problemáticos gramaticales.

3. En cuanto a la organización de los intercambios, sugirieron que hubiera un mayor compromiso entre ambas partes y un calendario más estricto y puntual entre los comunicados.

4. Otros comentarios se refieren a la edad e intereses de los alumnos canadienses, ya que las características de éstos eran muy diferentes a las de mis estudiantes, la mayoría de los cuales — como se ha señalado ya líneas arriba - son muy jóvenes y casi ninguno de ellos tiene aún experiencia en el mundo laboral, perfil muy diferente al de los estudiantes canadienses, cuyas edades fluctuaban entre los 40 y 60 años y todos eran profesionistas.

Para concluir, he de decir que, personalmente, la experiencia ha sido muy grata y muy enriquecedora. Aun cuando no tuve comunicación directa con ninguno de los estudiantes canadienses, sí tuve la oportunidad de revisar todos sus correos para poder elaborar la sistematización de errores.

En este sentido, aunque por mi experiencia como profesora podía prever los errores gramaticales que encontraría - y me refiero, principalmente, al conocimiento de los aspectos gramaticales más problemáticos que enfrenta un francohablante que quiere apropiarse del español-, he podido observar el correcto tratamiento sobre los mismos por parte de la profesora Emma Jiménez que ha sabido encontrar nuevos caminos para evitar la fosilización de dichos errores, pues varios de éstos que aparecían insistentemente en los primeros correos que recibimos, ya no aparecieron en posteriores comunicados.

Finalmente, creo que esta experiencia debe continuar por lo enriquecedor de sus primeros resultados para todos los que tuvimos la oportunidad de participar en ella. Además, no dudo que, con los comentarios, sugerencias y participación de la comunidad de profesores de nuestro Centro, esta experiencia pueda mejorarse y llegar tan lejos como lo permitan nuestra imaginación y nuestro esfuerzo. 


\section{SISTEMATIZACIÓN DE ERRORES DE LOS ALUMNOS DE LA ESECA. PROYECTO CEPE-ESECA.}

Marzo-mayo del 2000

I. MORFOLOGÍA

\section{$\underline{\text { Verbo }}$}

a. Errores de tiempo/modo.

a.1. Uso del pretérito de subjuntivo por el pospretérito:

Ej.: ¿Pudieras mandarme una foto de tu casa? (JU)* ¿Pudiera ud. describirme...? (JU)

a.2. Pospretérito por pretérito de subjuntivo.

En este caso, el problema parece estar en el desconocimiento de los usos verbales para las condicionales hipotéticas en presente:

Ej.: Si usted querría compartir conmigo... (FRAN)

Si ud. querría saber más de la vida en... (FRAN)

a.3. En ciertas construcciones de relativo, hay algunos ejemplos de confusión de pretérito de subjuntivo por pospretérito:

Ej.: ...para satisfacerlo con una que le gustaría... (JU)

\section{b. Errores de modo indicativo/subjuntivo}

Ej.: ¿Pudiera describirme el modo de vida en que viva usted? (JU) En muchos casos, parece tener relación con el manejo de las oraciones de relativo.

Espero que tienes... (CYNTHIA)

... hecho que demuestre al ser humano... (JU)

• Los nombres que aparecen entre paréntesis, corresponden a los alumnos canadienses. 
c. Confusión de ser/estar.

Ej.: ¿Dé dónde está usted? (JU)

... de temperatura fría, como lo está en Canadá... (FRAN)

Cuando seré jubilada. (MICHEL)

... soy $(i)$ - sic — en carga de... (CYNTHIA)

d. Confusión de reflexivos y falsos reflexivos.

Ej.: $\quad$ Se comprobaron ser mis mejores amigos. (JU)

..._envejeciéndose (una persona)... (JU)

... y mejorarme en conversación... (REX)

Quiero perfeccionarme (mi español)... (DIANE)

e. Confusión de futuro de indicativo por presente de subjuntivo en las oraciones subordinadas temporales futuras.

Ej.: Cuando seré jubilada. (MICHEL)

f. Errores de conjugación:

f.1. Pretérito: (Yo) Vive ocho agnos... (ERIC)

(Yo) Me fue de compras... (JU)

Ya había pasado 40 años... (JU)

(Yo) hubo de pararme muchas... (JU)

g. Confusión de infinitivo antecedido por la preposición “a”, por gerundio.

Ej.: ... paso mucho tiempo a bailar, cenar,...

Si solamente podía pasar mi vida a viajar. (CYNTHIA)

h. Confusión de copretérito por pretérito de subjuntivo.

Si solamente podía pasar mi vida a viajar. (CYNTHIA)

i. Confusión estar/tener en la perífrasis obligativa tener que.

Ej.: ... estuve que viajar a Europa... (JU) 


\section{Verbos de construcción invertida}

a. Falta de concordancia entre el verbo y el sujeto sintáctico.

Ej.: Me fascina la historia y las civilizaciones... (REX)

b. Confusión de los sujetos sintáctico y semántico (omisión de a).

Ej.: ¿Qué le gusta usted hacer...? (CYNTHIA)

\section{Preposiciones}

a. a/en

Ej.: ...enseña usted a la universidad... (FRANCINE)

...empecé a tomar cursos a la UNAM... (FRAN)

...empecé a trabajar a la Oficina... (MICHEL)

...tomando cursos a la UNAM... (CYNTHIA)

...trabajo a la Comisión... (CYNTHIA)

...de compras a una librería... (JU)

b. A/para.

Ej.: ...que sea beneficiosa a la escuela y a mí mismo (REX)

c. Uso superfluo de la preposición "de" en ciertas subordinadas con el mismo sujeto.

Ej.: $\quad$ Espero de encontrar la oportunidad... (REX)

Será un placer de darle... (FRAN)

Estoy planificando de mudarme... (CYNTHIA)

Mi sueño es de conocer la Rusia... (CYNTHIA)

\section{d. Para/por}

Ej.: Trabajé en Argentina para otro 4 meses... (CYNTHIA)

\section{e. De/por}

Mal manejo de la estructura de la voz pasiva.

Ej.: Fui licenciada en historia de la universidad de... (MICHEL) ${ }^{* *}$

f. Uso innecesario de la preposición "a" con objetos directos que no son personas o no se pueden personalizar.

Ej.: $\quad$...alcanzó al corazón de los hombres... (JU)

...él reinaba cuidando al terreno y a la única flor... (JU)

...los principios que rigen a toda sus existencias... (JU)

** Esta alumna tiene problemas con el manejo de la estructura de la pasiva plena. 
g. Omisión de la preposición "a" para objeto directo de persona.

Ej.: ... dejando (El Principito) el aviador más triste... (JU)

\section{Pronombres personales}

a. Problemas de mala declinación.

a.1. Reflexivo.

Ej.: Considere satisfactoria para yo mismo (REX) Sobre yo mismo (ERIC)

a.2. Uso del dativo (le) por el acusativo (lo)

Ej.: Es interesante notar que le escribió... (JU) ('le' sustituye a el libro)

b. Confusión de los registros informal (tú) y formal (ud.)

Ej.: Espero que tienes tiempo y pronto reciba su e-mail. (CYNTHIA)

\section{Concordancia}

\section{a. Sustantivo-adjetivo}

\section{a.1. Género:}

...conversación español. (REX)

...otro parte... (FRANCINE)

...ciudad peligroso or tranquilo... (FRANCINE)

...música latino... (FRAN)

...los danzas... (FRAN)

Mi padre era suiza... (ERIC)

...en la planeta...; la pequeña planeta... (JU)

...estilo pesimisto... (JU)

...la asma... (JU)

\section{a.2. Número:}

...fuentes local.. (REX)

...ruinas histórica... (REX)

En los años 1970... (MICHEL)

Trabajé en Argentina para otro 4 meses... (CYNTHIA)

Otro símbolo fuerte, el del elefante..., el pequeño cordero... (JU) 


\section{SINTAXIS}

En este rubro, la sistematización queda incompleta, dado que el nivel de los estudiantes que se prestaron a esta aventura es intermedio y, cuando tratan de expresar ideas más complejas, enfrentan muchos problemas con las subordinadas, principalmente, con la concordancia tanto modal (indicativo/subjuntivo) como temporal.

Sin embargo, los errores más frecuentes y generales y que pueden ser ya trabajados en clase se refieren, tanto a las oraciones subordinadas de relativo como a las oraciones subordinadas temporales y finales.

\section{OTROS}

a. Confusión en las grafías ( ¿o no la tiene su computadora?): $\mathrm{GN}>\tilde{\mathrm{N}}$ agnos, espagnola, espagnol. (ERIC)

b. Faltas de acentos en general en todos los correos que pueden ser debidos, asimismo, a su inexistencia en las computadoras que ellos manejan.

c. Problemas de vocabulario y de uso de falsos cognados:

Ej.: exprimir por expresar, exponer.

destinación por destino.

tiempo por hora

chasón por canción.

alcanzar por ganar.

aparece por es evidente (que)

d. Problemas de puntuación que no sé si se deben al diferente uso de éstos en español, inglés y francés, o al desconocimiento de sus reglas por los alumnos. Me sería difícil señalarlos todos, pero los más frecuentes son los del punto y coma y los dos puntos. 


\section{UNIVERSIDAD NACIONAL AUTÓNOMA DE MÉXICO CENTRO DE ENSEÑANZA PARA EXTRANJEROS}

\section{Cuestionario para saber los resultados de los intercambios con Canadá Prof. CRISTINA Simón, coordinadora en el Distrito Federal}

1. El tiempo que dedicaste al intercambio fue:

Demasiado ( ) Razonable ( ) Muy poco ( )

Comentarios:

2. El número de intercambios que tuviste con tu destinatario fue:

Excesivo ( ) Razonable ( ) Poco ( )

Comentarios:

3. Los temas que se trataron en el intercambio fueron:

Interesantes ( ) Poco interesantes ( )

Comentarios:

4. ¿Incluirías otros temas en el intercambio?

Sí ( ) No ( )

¿Cuáles?

5. Este intercambio, ¿te sirvió para conocer mejor tu lengua?

Sí ( ) No ( )

¿Por qué?

6. ¿Y para darte cuenta de ciertos aspectos gramaticales problemáticos de la misma para un extranjero que pretende apropiarse de ella?

Sí ( ) No ( )

¿Por qué? 
7. En el plano de la comunicación cultural, ¿enfrentaste algún problema de entendimiento con tu destinatario?

Sí ( ) No ( )

¿Cuál(es)?

8. ¿Y en algún otro plano —de estrato social, de edad, de profesión—?

Sí ( ) No ( )

¿Cuál(es)?

9. ¿Crees que tu participación en este intercambio te pueda ayudar en un futuro a ser mejor profesor(a) de español L2/LE?

Sí ( ) No ( )

¿Por qué?

10. En cuanto a tu colaboración para la revista de la red —Flores de Nieve-, ¿escribiste el artículo?

Sí ( ) No ( )

¿Por qué?

11. ¿Te habría sido más fácil participar si se hubiera propuesto un tema concreto?

Sí ( ) No ( )

¿Por qué?

12. Si tu respuesta fue afirmativa, ¿te costó mucho esfuerzo escribirlo?

Sí ( ) No ( )

¿Por qué?

13. Los comentarios de tu destinatario, ¿te ayudaron a mejorarlo?

Sí ( ) No ( )

¿Por qué?

14. Comentarios y sugerencias: 